\title{
Construction of China's Publishing Enterprise Agile Supply Chain
}

\author{
Mei juan Zhang ${ }^{1}$, Yun $\mathrm{Cai}^{2}$, Xiang Hui Yang ${ }^{2}$ \\ 1 Zhang Mei Juan, Dr., associate professor, School of Information \\ Management of Wuhan University, 430072 Wuhan, Hubei Province, \\ P.R.China. email: sqzmj@126.com \\ 2 Postgraduates, School of Information Management of Wuhan \\ University, 430072 Wuhan, Hubei Province, P.R.China.
}

\begin{abstract}
China's publishing enterprise Agile Supply Chain has its own peculiarities. The authors elaborate the significance of building the Chinese publishing enterprise Agile Supply Chain based on dynamic alliance in the current highly competitive market, and then construct and analyze three models and their characteristics. At last the authors discuss some problems deriving from the information Game, compatibility of enterprise knowledge and culture, and the administrative structure among Supply Chain member enterprises when the Agile Supply Chain comes into practice. .
\end{abstract}

\section{Introduction}

It's known to all that in recent years competition among the enterprises has gradually been evolved to the competition among supply chain. In the rapidly changing market environment, the competitiveness of supply chain mainly embodies in a prompt response to customer demand and turns it into a commercial opportunity.

Compared with traditional supply chain, Agile Supply Chain is an organizational strategic "alliance" which lay much stress on adapting itself to the market "dynamic" changes. It drives on the core enterprise, and with the control of logistics, capital flow, information flow, integrates suppliers, manufacturers, distributors, retailers and end-consumers to an integrated functional network chain[1]. Its main features are: (1) Market Sensitivity. This is an essential feature for Agile Supply Chain which can deconstruct and reconstruct smoothly in a timely manner so as to meet the changing market demands. (2)Process Integration. General supply chain focuses on information exchange and sharing, while Agile Supply Chain integrates business process across enterprises. (3) Network Structure. Agile Supply Chain integrate geographically dispersed

Please use the following format when citing this chapter:

Zhang, M., Cai, Y., Yang, X., 2007, in IfIP International Federation for In Cormation Processing, Volume 251, Integration and Innovation Orient to E-Society Volumel, Wang, W. (Fds), (Boston: Springer), pp. 371-378. 
enterprises based on the business process integration, supply cooperation, coordination, remodeling and distribution of interests among chain partners, rather than a single vertical "pyramid" structure.

\section{The Significance of Building Publishing Enterprise Agile Supply Chain}

\subsection{Latest Changes of Book Consumer Market in China}

With the rapid development of Chinese economy and new technologies and ideas which have been constantly introduced, the demand of reader for knowledge is of increasing diversification and customization. The fact that book consumers keep changing entirely and rapidly shows as follows: (1)Book consumer market turns from popularization to customization, needing a more variety of book distribution with less quantity and more frequency. (2)The lifecycle of book market is getting shorter. On the market-oriented production mode, one should be able to capture the market opportunities when it could meet the consumption market needs in the highest speed, which makes a new challenge for publishing enterprise Agile Supply Chains[2].

\subsection{Major Problems in the Existing Publishing Enterprise Supply Chain}

The publishing enterprise supply chain process mostly covers production, circulation and marketing with the aim at increasing the sales, decreasing the costs and gaining the profits. In the new marketing environment, there are some problems in the actual publishing supply chain. They are as follows.

(1) On the whole, Chinese publishing industry supply chain models can be classified into two major categories, publishing group supply chain and SMEs supply chain. The former operation is a basic administrative ties and a "pyramid" business organizational model, and its structure and function are too "rigid" and rarely adapt to the market "dynamic" nature. The latter one is the strength of enterprises mixed and lack of effective platform and linkage mechanism. So, the operation appears to be too "loose" rather than "alliance". Both of the supply chains have failed to achieve expected efficiency and to satisfy to the demands of market.

(2) The existing publishing enterprise supply chain runs by taking production rather than market as a starting point. In the case, the downstream enterprises being in a passive position usually increase their stocks to meet variable demand, which causes the growing inventory and poor response to the demand changes of readers.

(3)Information sharing mechanism and operational cooperation mechanism between enterprises don't work well. 
An authoritative statistics[3] shows that in the year of 2005, the total sales of publications of the state-owned distributors are 15.798 billion lists (sheets, shares, boxes), up to 122.981 billion PMB yuan, increasing by $2.02 \%$ and $7.52 \%$ respectively in quantity and money compared with the performance last year, but the inventory grows much up by $26.9 \%$ and $39.3 \%$ respectively. The situation shows that publishing enterprise Agile Supply Chain should be built immediately to optimize and integrate resources and fit in with the changes of market.

\section{Construction of Publishing Enterprise Agile Supply Chain}

\subsection{The Basic Principles of Construction of Publishing Enterprise Agile Supply Chain}

For the majority of enterprises, including publishing enterprise Agile Supply Chains, Agile Supply Chain is a new concept. Meanwhile, as a content industry, Chinese publishing industry has its own specific characteristics. So, in order to construct successfully the publishing enterprise Agile Supply Chain in China, the following basic principles should be adhered to, apart from general principles [4] such as standardization and enterprise win-win, etc.

(1)Core enterprise (or leading enterprise) must be established in the proposed Agile Supply Chain. Without the core enterprise, the whole supply chain will be of unsubstantial operation because of no force of organization and drive. This core business generally is a strong competitor or one with certain competitive advantages.

(2)The focus concerning Agile Supply Chain Integration is business process of the product chain rather than enterprises. Only by reasonable regulation on the course of business can the enterprise integrate and utilize internal and external resources. So, all major business links and process of production should be decomposed in management from the perspective of industrial chain, and should learn about the information of operating entity with higher level of business specialization.

(3) With the core business' organization, an opening platform for information exchange and relative stability system facing a supply chain need to be established. Just like a printed circuit board, its architecture provides a slot standard and agreement between the slots link, so qualified components can be placed in slots. This assembly system of production, the use of open standards, the autonomy of "building blocks", sharing extensively common functions, Plug compatibility, allowing heterogeneous environment, the self-learning modules and self-organizing capacity, achieve reconstruction rapidly which feature Agile Supply Chain requires.

(4) The relevant publication policy should also be considered when building the publishing enterprise Agile Supply Chain. For example, the production of books in China is not allowed to run by the non-state-owned entities yet, thus, the non-state-owned entities should not be included in the publishing enterprise Agile Supply Chain. 


\subsection{Three Types of Publishing Enterprise Agile Supply Chain}

Based on the actual situation of China's publishing industry, we have divided the publishing enterprise Agile Supply Chain into 3 types with the analysis of their characteristics from the perspective of industrial chain based on the decomposition of publishing process.

\subsubsection{The Models and Features of Publishing Enterprise Agile Supply Chain Taking Publisher as the Core Enterprise}

Some of the powerful publishing groups, such as the Chinese publishing Group, the Shanghai Century Publishing Group and Liao Ning Publishing Group, and other production-based book publishing enterprises could establish the publishing enterprise Agile Supply Chain taking publishers as the core enterprise, the model is shown in figure 1.

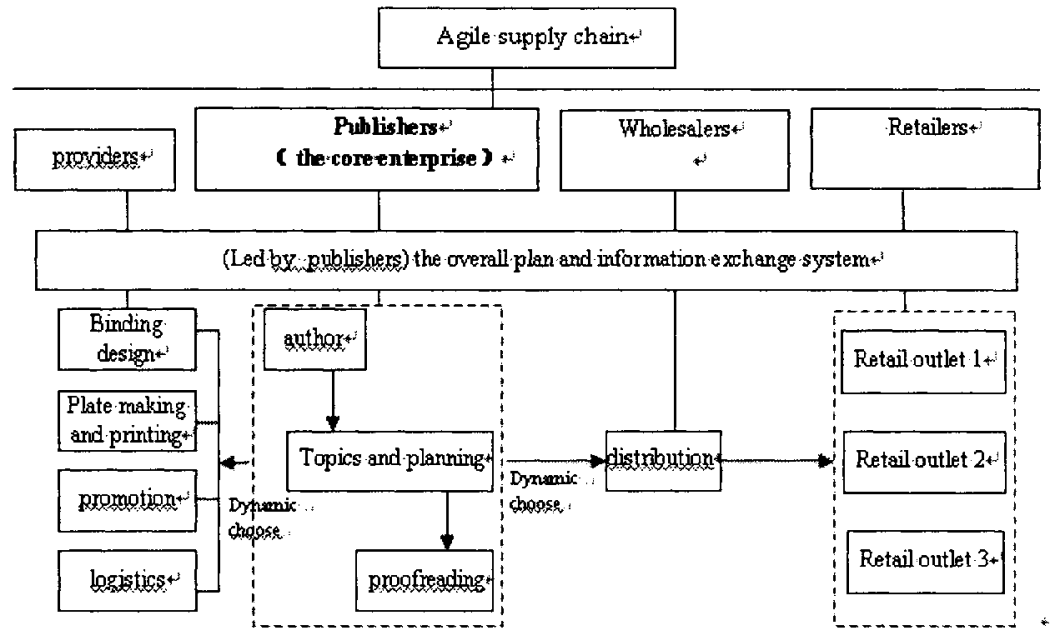

Fig. 1. the model of publishing enterprise Agile Supply Chain taking publishers as the core enterprise

These publishers could use its outstanding content production to attract resources. They could choose the right product wholesalers and retailers according to the nature of their product through contracts. They could also outsource their non-core business through contract, such as Binding design, plate making and printing, logistics, etc. in order to achieve optimal allocation of resources and to establish a strategic cooperation relationship. At the same time, they are responsible for the establishment and maintenance of the supply chain and information exchange platform. 


\subsubsection{The Model and Features of Publishing Enterprise Agile Supply Chain Taking Wholesalers as the Core Enterprise}

The distributing groups of Xinhua Bookstore and the private wholesales have been well developed in recent years. It lays a good foundation for establishing the publishing enterprise Agile Supply Chain taking wholesalers as the core enterprise. The model is shown in figure 2.

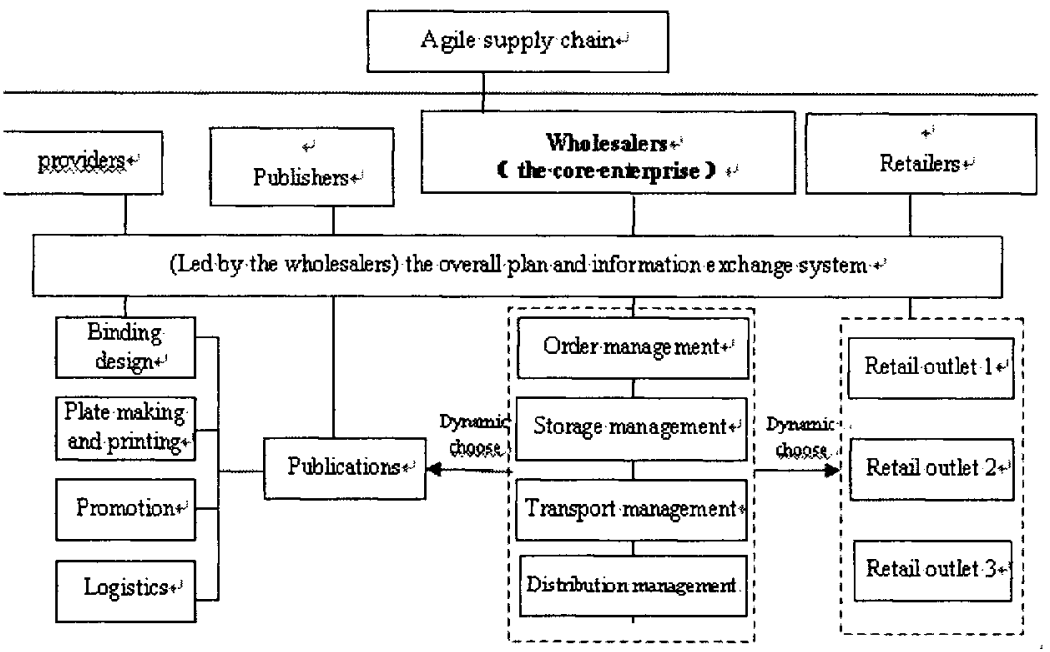

Fig. 2. the model of publishing enterprise Agile Supply Chain taking wholesalers as the core enterprise

The main features of this type of Agile Supply Chain are: (1) The wholesalers lead the Agile Supply Chain planning and the overall information-sharing system. They provide the real-time information about the distribution of publications to the other members on the chain. And they are in charge of the maintenance and management of information exchange in the entire supply chain. (2) Locating in the middle of the chain, wholesalers have the advantage of connecting the upper and lower compared with the other two types of supply chain. So the operation of this type of supply chain should be relatively easier. (3) The wholesalers select the appropriate upstream and downstream enterprises according to different publications in order to find the best and fastest way to deliver the publications to the retail outlet through various forms of dynamic alliance.

\subsubsection{The Model and Features of Publishing Enterprise Agile Supply Chain Taking Retailers as the Core Enterprise}

With the development of market-oriented production business model and the formation of "terminal is King" principle, the books retail terminals and large bookstores develop rapidly, such as Shenzhen Bookmall, Beijing Book Building. These retailers could and need choose their own channels and wholesalers under 
its own strategy, namely, to establish the publishing enterprise Agile Supply Chain taking retailers as the core enterprise. The model is shown in figure 3.

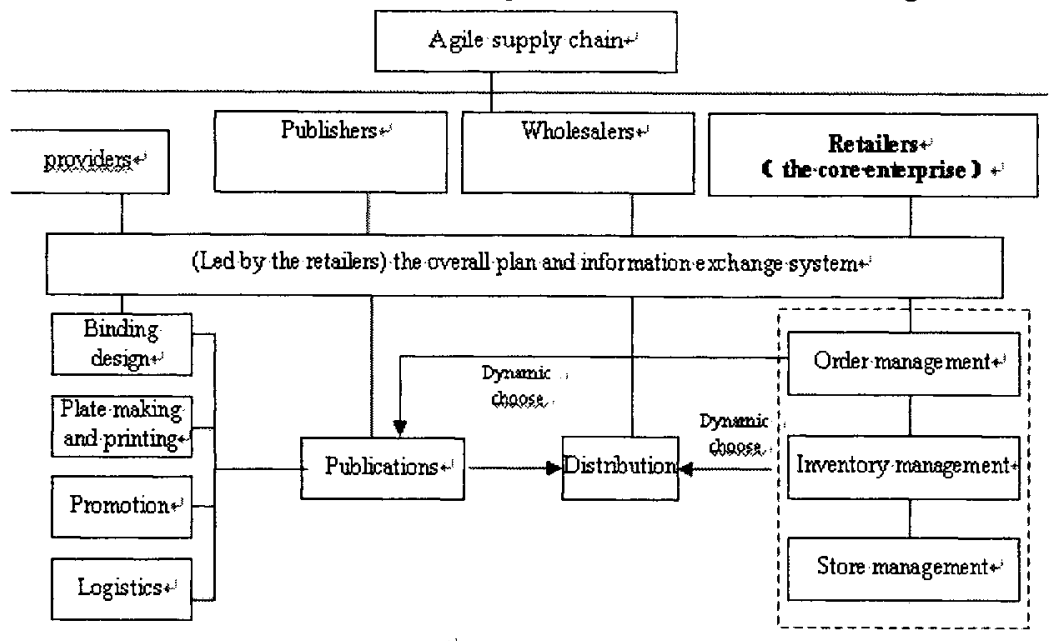

Fig. 3. the model of publishing enterprise Agile Supply Chain taking retailers as the core enterprise

The main features of this type are: (1) The retailers lead the Agile Supply Chain planning and the overall information-sharing system. They provide the real-time information about the sales of publications and the demand of readers to the other members on the chain. And they are in charge of the maintenance and management of information exchange in the entire supply chain. (2) The retailers select the appropriate upstream and downstream enterprises according to the sales of publications in order to quickly respond to book market and make every effort to achieve the ultimate goal of " seek readers for publishers, seek books for readers " through various forms of dynamic alliance

\section{Issues which should be Paid Attention to when Operating the Publishing Enterprise Agile Supply Chain}

\subsection{The Effectiveness of Information Transfer}

The immediate, accurate and complete information transfer amongst enterprises is an important node of normal operational support to Agile Supply Chain. However, as the members of the supply chain enterprises have their own independent interests and multiple purposes when joining the supply chain, the contract alone may not address differences in the conflict caused by interests. So the information transmission of the supply chain could be easily delayed and distorted. On the other hand, because of the information asymmetry in the actual information transmission process, the information senders occupy the authentic information so that the information receiver couldn't grasp, which makes the Game of information act in the process of information transfer is inevitable. That means the information senders will send information for their own benefit, and the information receivers will try to take the real messages hidden behind it. 
Take the supply chain of wholesalers and bookstores for example. If the bookstore hides the demand information from wholesalers, it will cause inventory backlog to wholesalers. And if the wholesalers keep their own distribution capabilities and circulation progress as confidential information, the arrival time and the quality of books for bookstore will be affected. In other words, as for the downstream enterprises on the publishing supply chain, every enterprise is reluctant to share their information. But to the upstream node enterprises, the more the knowledge they share, the greater the profit they will get. Therefore, upstream enterprises are willing to share their information with all enterprises on the supply chain. Obviously, this information Game will affect the effectiveness in information transfer.

Building a risk sharing and incentive mechanisms based on economic interest between enterprises in the supply chain is a fundamental measure, which can reduce the information asymmetry, and can effectively prevent the negative impact of information Game. There is an incentive mechanism that the adverse side will get some compensation in order to promote the cooperation. And the balance of the information Game could be implemented by the punishment to non-cooperation betrayal [5].

\subsection{The Cultural and Knowledge Coherence among Member Enterprises}

Besides the complete sharing of information, the success of Agile Supply Chain also relies on the exchange of knowledge and resources between the member enterprises, which could enhance competition in the multi-joint and avoid short board phenomenon. However, the access to resources and knowledge is particularly relying on the compatibility of the strategy and knowledge of different member enterprises. In selecting a strategic partnership, if the two are at quite different levels of knowledge, the receiver is unable to accurately understand and absorb knowledge, there will be obstacles in information exchange and the dynamic union will lose its efficiency.

In addition, each business has its own unique corporate culture. In Agile Supply Chain, the compatibility of enterprise culture also has a profound impact on the success of dynamic union. The high coherence of cultural and strategy brings the high efficiency to the strategic alliance. Therefore, when choosing the member enterprises on supply chain, we should also consider the coordination of the corporate culture of the member enterprises on the same chain.

\subsection{The Administrative Compatibility of the Members Enterprises}

Chinese current publishing distribution system is based on administrative region. Its vertically integrated supply chain operations have gradually formed fixed groups of interest. How to break the internal structure of the interests of the system, to eliminate various practical differences, to realize the actual supply chain system based on enterprise efficiency and effectiveness itself turns out to be important issues which should be seriously taken into consideration when we are aiming at building China's publishing enterprise Agile Supply Chain and dynamic alliance. 


\section{References}

1. Z.F. Wang, Supply Chain Management, Publishing House of Electronic Industry, Beijing, 2006, p.26.

2. Z.H. Qu, Five Changes in Chinese, Publishing Market, March 6, 2007;

http://ent.sina.com.cn/x/2006-05-05/08561072682.html

3. China Publishing Yearbook2006. Publishing House of China Publishing Yearbook, Beijing, 2006), p.731

4. C.Y Gao, Analysis on the Supply Chain of Publishing Group, A Vast View on Publishing, 2004 (10)

5. J.N. Wu, B.L. Liu, Analysis on Supply Chain Strategic Partnership with Game theory, Logistics Technology, 2004(4) 